was above, for on turning the patient to better wash the cavity the adherent diaphragmatic and parietal pleura suddenly broke apart and air and pus rushed into the pleural cavity; the lung collapsed, leaving the diaphragm like a flapping sail between the pleural and the abscess cavity. After washing both thoroughly with salt solution, I endeavored to close off the pleural cavity by gauze packing and drained the subdiaphragmatic space with tubes.

The patient bore the operation well, but was quite cyanotic during the night. In the morning his condition seemed fair, but distinctly worse than the night before. His face was anxious with the peritonitic expression. After dressing his chest wound I found that his abdomen was distended and somewhat tender, though he complained of no pain in it. There was, however, marked rigidity of the muscles so characteristic of peritonitis. I came to the conclusion that in my manipulations of the subdiaphragmatic abscess I must have ruptured it on the abdominal side, and by syringing spread the contents through the abdomen. It seemed that there was one possible chance of making an early diagnosis of this and once more flushing out the abdominal cavity. After giving it a careful study, I explored the left side of the abdomen under cocaine in the semilunar line. I found the abdomen full of creamy pus. Giving the patient a little ether I enlarged the incision and washed out the whole peritoneal cavity, except that part walled off by adhesions, and having put cigarette wicks in the splenic, pyloric and pelvic regions, partially closed my incision.

For five days after this the patient was in a very critical condition, barely able to stand the dressings which were necessary to remove the immense quantity of discharge from his peritoneum, pleura and subdiaphragmatic space.

On the seventh day, i. e., just a month after the original operation, he seemed to be fairly out of the woods, when his temperature began to rise again. For several days the same rise continued, without pain or demonstrable local tenderness. On the tenth day a doughy mass hardly distinguishable from the right kidney began to appear, and by the fourteenth day was so well marked that I decided to explore it.

Under cocaine I made an incision in the flank and, to my dismay, entered the peritoneal cavity behind the adherent colon. The abscess could then be felt between my finger in the flank incision and the anterior abdominal wall. It was tympanitic in front and behind, due, as I afterwards found, to the ascending colon behind and to the outer side of it, and some coils of small intestine above and to the inner side of it. Following the peritoneum downward I found no pus, but on going upward toward the liver I broke into a pocket which also extended down to the right of my original wound toward the pelvis. This I cleansed thoroughly with gauze and drained.

Between this operation and the first one, the patient's bowels had been quite loose, about four movements in the twenty-four hours.

On Oct. 4, he was improving and went out on the veranda in a stretcher. The sinuses were doing well. On Oct. 5, he had another attack of severe abdominal pain, and the surgeon in attendance explored the left side of the abdomen under cocaine. A large amount of clear fluid was found and the transverse colon appeared the size of a lead pencil. It was considered that there must be an obstruction in the hepatic flexure, so another opening was made over the cecum and a Mixter tube inserted. During the day there was no escape of gas or feces from this opening, so that on the next day another operation was done and a band was found which obstructed the small intestine. This band was removed and another Mixter tube tied into the small bowel. Following this operation the obstruction was relieved, but the patient's strength failed to such a degree that he died on the tenth day following, about eight weeks from the time of perforation.

Perforation, Aug. 22, 1903; first operation, Aug. 23; wicks out and changed and shortened, Aug. 27; second operation, Sept. 12; third operation, Sept. 13; fourth operation, Sept. 27; autopsy, Oct. 17, 1903 (No. 1132). Anatomical diagnosis. - General fibrinopurulent peritonitis; subphrenic abscess; purulent bronchitis; abscesses of the lungs; empyema; ulcer of the duodenum; edema of the kidneys.

In the wall of the duodenum, antero-laterally and to the right, there are several strands of suture material. On the passage of water, under pressure, through the stomach and the duodenum, the sutures hold, and there is no leakage from the duodenum. On cutting the sutures and then laying the stomach and duodenum open there is seen in the mucosa of the duodenum, at a point beneath the situation of the sutures, an ovoid loss of substance about $6 \mathrm{~mm}$. in greatest dimension, with smooth rounded edges and a base which is very thin and membranous in character. In the central portion of this membranous base there is a small opening about $32 \mathrm{~mm}$. in greatest dimension. The mucosa of the intestines elsewhere presents nothing worthy of note.

(To be continued.)

\section{POINTS IN THE DIAGNOSIS OF INJURIES.}

\section{BY F. J. COTTON, M.D.,} Assistant Professor in Clinical Surgery, Tufts College Medical School;
Assistant Surgeon, Boston City Hospital.

Definite, positive single "points" are often of the greatest value, both in medicine and surgery, whether they are actually the direct means of establishing a diagnosis or merely the signpost that puts us on the right road.

A few such points are here presented. All are the result of personal experience; all confirmed by repeated observation.

Some of them have no doubt been observed before; most of them I believe to be new. ${ }^{1}$ The detailed case-histories and reports will not be given here, in part because notes were often taken only in briefest fashion at the time, in part because the later following up of hospital cases, in such a way as to be able to give full reports, calls for an expenditure of time not now at my command.

Moreover, the value of observations of this sort must always depend, in the end, upon the testing of the individual conclusions of one observer by the " checking" observations of other observers, in the nature of things less interested observers, and probably, therefore, more impartial.

I give the following conclusions for what they are worth, adding only that I believe each of definite value and have tested each, not only repeatedly, but through a course of several years on a considerable clinical material.

\section{SHOULDER LUXATION.}

After luxation of the shoulder, of subcoracoid or subglenoid type, there are two points that show localized tenderness to pressure, and to which

1 That is, new except as I have mentioned them, en passant, in That is,
other articles. 
much of the pain of motion is referred. The first is a point slightly below the projection backward (behind the acromion) where the scapular spine and the acromion come together. At this point there is usually a sharp spur. The point of tenderness lies just below this spur. The second point is in the region of the coracoid process itself.

Tenderness at these points occurs sometimes with simple "sprains" of the shoulder, but is in some measure confirmatory of the diagnosis of a (reduced) dislocation. So far as my observation goes, absence of such tenderness, at least of that below the scapular spine, renders the diagnosis of such a reduced dislocation very doubtful. Presumably these points of soreness correspond to ligament damage; certainly they correspond to the major ligament insertions.

\section{SHOULDER INJURIES.}

In injuries of the shoulder, particularly after luxation, there is often during convalescence a consistent complaint of pain referred to the region of the deltoid insertion on the humerus. Sometimes, but not as a rule, there is sensitiveness on pressure at this point. I have not been able to make any definite use of this point in diagnosis, save that it seems more common in luxation than in the shoulder fractures. It has no obvious bearing on final prognosis of usefulness.

BICEPS RUPTURE (RUPTURE OF THE LONG HEAD)

This is not the rare injury that one might suppose from the literature. Favored by the atrophic and erosive lesions of chronic arthritis, it occurs often with but slight trauma; most of those cases seen are in joints that we can hardly call normal.

The signs given for diagnosis, usually, are the pain on active supination or passive pronation of the flexed forearm, but besides these, we have:

(a) A slight forward displacement of the humeral head, due to the lack of the restraint normally exercised by the tendon as it crosses the joint.

(b) The characteristic deformity of the muscle on attempted flexion, a bunching of muscle below the break $(A$, Fig. 1) and an abnormal hollow above. (B, Fig. 1.)

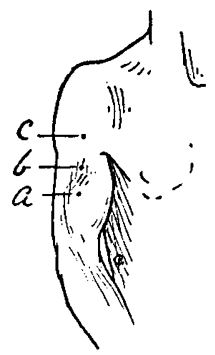

Frg. 1. Biceps rupture. $a$, bunching of muscle below the tear $b$, hollow above the tear; $c$, loss of resistance on palpation, also the site of ecchymosis.

(c) Tenderness along the course of the long head.

(d) Ecchymosis along the same course.

(e) Disappearance of the long head on palpation.

On comparison with the sound arm, we may very readily feel the absence of this tense tendinous origin on active resisted flexion. This is felt at the point marked $C$ in Fig. 1.

\section{ELBOW FRACTURES.}

Loss of supination and pronation (with extension and flexion normal or nearly so) means damage, not to the humerus, but to the neck or head of the radius. ${ }^{2}$

Loss or limitation of flexion and extension (with rotation undisturbed) means damage to the humerus, not to the bones of the forearm.

Loss of flexion alone (with the sharp check obviously due to contact of bones on bone) can only result (1) from supracondylar fracture with forward displacement of the shaft; (2) from supracondylar fracture with rotation about a vertical axis; (3) from luxation of both bones of the arm backward; (4) from luxation of the radius forward.

Loss of extension alone may mean a supracondylar fracture with forward displacement of the lower fragment (Kocher's "supracondylar from flexion "), or more commonly it may mean a chip of bone (oftenest from the face of the external condyle ${ }^{3}$ ) getting in the way in the joint (between olecranon and either condyle) like a stone in a hinge, or in the front of the joint acting similarly. A hollow directly above the olecranon behind can mean only a backward luxation; a hollow slightly higher means supracondylar fracture. In the luxation the hollow of the sigmoid cavity may be palpable; in the fracture the abnormal mobility forward and back is little limited, and crepitus is usually obtainable.

Pain limited to passive flexion or active extension means damage to the olecranon - either a fracture of the bone or (rarely) an epiphyseal separation. ${ }^{4}$

Marked lateral deviation of the forearm means supracondylar fracture or total epiphyseal separation (or possibly a lateral luxation, which is relatively rare). It does not occur with fractures of either external or internal condyle, despite the great mass of literature, beginning with Allis, alleging that it does occur. ${ }^{5}$

Elbow landmarks. The classical picture of the elbow landmarks is false. The two condyles and the tip of the olecranon do not form an equilateral triangle in any position. The internal condyle is distinctly nearer the olecranon than is the external.

Lameness, ecchymosis and, later, atrophy (often very sharply marked) along the course of the flexor and pronator muscles having their origin in the internal condyle of the humerus indicate either fracture of the internal epicondyle ${ }^{\circ}$ or severe strain affecting these muscles or the tendinous insertion of their origin from the elbow.

${ }^{2}$ I cannot agree with Mouchet, who finds such limitation indicative of damage to the external condyle. In external condyle fractures I have found it but once in about thirty such cases examined with this point in view. In fractures of radial head or neck there seems to be a constant limitation of rotation, usually permanent. ${ }^{3}$ Cotton and Sylvester: Fracture of the Capitellum. Boston MED. AND SuRg. Jour., Dec. 31, 1903, cxlix, p. 734-6.

400. Cotton: Separation of Olecranon Epiphysis. Ibrid., June 28, ${ }^{5}$ Cotton: Ann. Surg., February, 1902

6 Cotton: Ibid.; Mouchet: Loc. cit. 
The most constant and recognizable landmark at the elbow is the ulnar nerve. ${ }^{7}$

\section{WRIST.}

Tenderness on pressure on the radius above the joint is significant only if it extends round about the circumference of the bone. If it does, that is proof presumptive (only negatived by a good $\mathrm{x}$-ray) that we have a cracked radius without displacement, such as we see to-day in the "automobile" fracture, and also in injuries from many other causes. Mere tenderness at one point on the circumference proves nothing. Fractures with displacement are, of course, to be recognized by the grosser tests, commonly described.

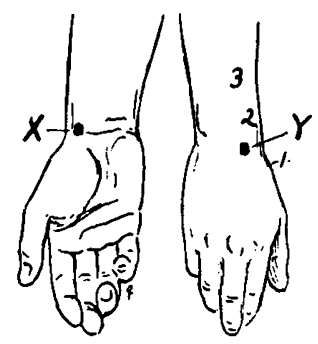

FIG. 2. 1, 2, 3, region of tenderness to pressure in tenosynovitis of thumb tendons. $X$ and $Y$, sites of tenderness, of ten with thickening, in scaphoid fracture.

"Crepitus" due to tenosynovitis of the thumb extensors (1, 2, 3, Fig. 2) almost precludes fracture or sprain; the tenosynovitis is (almost invariably, I think) due to the repeated slight trauma of overwork, a trauma which is at no single moment adequate to produce fracture or even sprain.

Tenderness on pressure over the sheaths of the thumb tendons, or effusion of blood and serum into their sheaths, may accompany wrist fracture, but such swelling and tenderness from a single trauma is not followed by a tenosynovitis that will give grating on motion of the tendon.

Loss of extension and of radial abduction at the wrist, with other motions free, suggest fracture or luxation of carpal bones. Ordinarily we are dependent for confirmation of this diagnosis on the $\mathrm{x}$-ray.

Tenderness on pressure over the radial side of the carpus, front and back, at the points shown as $X$ and $Y$ in Fig. 2, suggest scaphoid fracture but do not prove it, even if there is limitation of motion in the wrist. X-ray confirmation is usually essential.

RIBS.

Tenderness of the ribs alone is not a reliable sign of fracture; to be of any use as a sign the tenderness must be sharply localized at a definite point, and must be accompanied (in my belief) by crepitus, or at least by pain referred to this one point on pressure being exerted on the rib at some distance away. Contusions from direct blows in which there is no evidence of even a crack of the rib, are common. Possibly some of these are fractures, but we have no real ground for making the diagnosis.

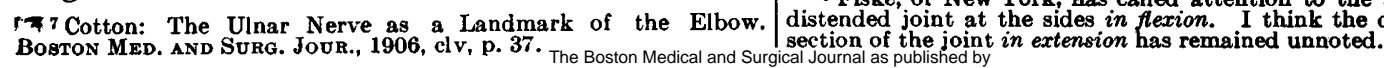
The New England Journal of Medicine. Downloaded from nejm.org at SANTA BARBARA (UCSB) on June 26, 2016. For personal use only. No other uses without permission. From the NEJM Archive. Copyright @ 2010 Massachusetts Medical Society.
Sharp pain toward the latter part of expiration, pain limited to a single, sudden, very painful catch, is at least very strongly suggestive of fracture. Lame muscles, etc., are apt tol give pain on inspiration.

The presence of lung or pleural complications are of course suggestive of fracture - they do not prove it.

Tenderness alone means little or nothing if confined to the point struck. Spasm of muscles at the point str. ck is not necessarily important.

Spasm of rectus muscle or other muscles not directly damaged means damage underneath, with rare exceptions.

Spasm of loin muscles (with recti uninvolved, or less involved) means damage to kidney as a rule.

Spasm of the whole abdomen after trauma means, usually, the beginning of general infection from rupture of one of the hollow viscera, or at least free bleeding from one or another source into the unprotected peritoneal cavity.

Spasm of abdominal muscles, single or general, in cases of spinal injury in the dorsal or lumbar region, proves nothing as to intra-abdominal conditions; it may be, and frequently is, simply an expression of nerve-root irritation.

\section{KNEE.}

Fracture of the patella gives an even distention of the knee-joint, with flotation of the fragments and distention of tissues in front of the ruptured and open capsule. (Fig. 3, B.)

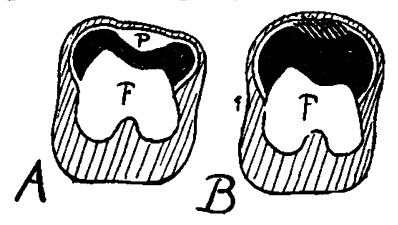

FIG. 3. Diagrammatic section of knee-joint in synovitis. The patella $(P)$ held down against the femur $(F)$ limits the upward expansion of effusion. $B$, like view in patella fracture; the patella is gone and there is no resistance to symmetrical, almost spherical, welling from effusion.

Synovitis of the knee gives a distention of the knee at the sides, limited in the median line by the intact patella and the extensor tendons. ${ }^{8}$ (Fig. 3, A.) In cases of an effusion so great as to make it impossible to feel the patella fragments, it is often of great use to have this differential sign, and I have not yet seen it fail in a considerable series of such cases.

ANKLE SPRAINS.

Almost all ankle sprains are sprains from inversion of the foot. In these sprains we often have considerable swelling, often with ecchymosis of the outer side of the foot. There are very often two points tender to pressure.

(a) The course of the peroneal tendons (see Fig. 4, A.)

(b) The anterior tibio-fibular ligament. There is in almost all sprains a point of especial, often ex-

8 Fiske, of New York, has called attention to the bulging of the distended joint at the sides in flexion. I think the different cross- 
treme, tenderness at a point shown as + in Fig. 4. In one case observed, this point afterward became the site of a moderate callus, persisting long after function was restored. The skiagraph showed only a slight roughening of the tibia at the ligamentous insertion. Evidently a small scale of bone had been torn loose. As a rule there is no such thickening; the lesion must be merely a tearing of ligamentous fibers. All that can be said is that this localized tenderness occurs almost constantly with the usual sprain; it has nothing to do any damage to the fibula and means nothing in prognosis.

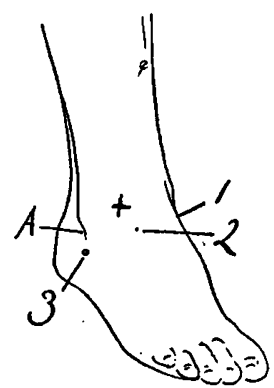

Fig. 4. $A$, peroneal tendons and anterior tibio-fibular ligament. 1,2 , neck of astragalus; 3 , outer surface of os calcis.

ANKLE LUXATION.

Inversion of the foot without shortening of the foot means (in the absence of well-marked malleolar fractures) luxation of the foot at the ankle, or beneath the astragalus.

Inversion of the foot with shortening means fracture or luxation farther down, usually in the medio-tarsal joint. Luxation in the medio-tarsal joint in the cases I have seen has given a shortening, measured from heel to the tip of the great toe, of nearly an inch. The visible deformity in these cases is curiously slight.

\section{ASTRAGaLUS.}

Swelling and tenderness at the points shown at 1 and 2, Fig. 4, suggests, especially if there is pain on dorsal flexion, a fracture of the neck of the astragalus.

\section{OS CALCIS.}

Thickening below the external malleolus on the outer side (Fig. 4, point 3) with disappearance of the projection of the malleolus, means comminuted fracture of the os calcis. ${ }^{9}$ This thickening is the result of the forcing outward of the outer cortical layer of the os calcis. Its bony character is usually clear enough; if there is doubt, massage and pressure will bring us down on unmistakable bone. I have never found this sign save in os calcis fracture, and have never found it absent in such fracture if of the comminuted type. Often the spreading of the bone is such as to give an entire effacement of the prominence of the external malleolus.

In this broadening that has been less precisely described as a "broadening of the heel." It is the reliable sign of the fracture, regularly present; $t$ e shoriening and raising of the heel are often not present in appreciable degree. ${ }^{10}$

${ }^{9}$ Cotton: Boston Med. ANd Surg. Jodr., 1906, clv, p. 645. 10 Cotton and Wilson: Ibid., October, 1908.
Loss of lateral motion of the foot with preservation of flexion and extension in the ankle usually means interference with the joints between astragalus and os calcis, not damage at the medio-tarsal joint. In default of obvious displacements this means, in the rule, a fracture of the os calcis, comminuted, involving this joint.

FOOT.

Localized tenderness in the region of the metatarsals means little; often there is no fracture.

Tenderness on pressure exerted on the ball of the foot, thrusting upward, in the line of the bone, on the metatarsal head, means trouble; as a rule it means metatarsal fracture.

Tenderness on lateral compression of the ball of the foot means little if confined to the region pressed on. If, however, pressure in this region gives pain, not at the point of pressure, but on the outer side of the foot higher up, it is presumptive proof of a fracture at this higher point. As met with in practise it is a usual sign of the fracture of the fifth metatarsal (at the base) by inversion, with which Jones, of Liverpool, has made us acquainted. ${ }^{11}$

The diagnostic signs here given are presented with some confidence in their essential accuracy, but subject, as hinted above, to confirmation by others.

VARIATIONS IN THE POSTERIOR HORN OF THE LATERAL VENTRICLE, WITH NOTES ON THEIR DEVELOPMENT, AND SUGGESTIONS AS TO THEIR CLINICAL SIGNIFICANCE.

BY E. J. CURRAN, M.D.,

Assistant in Anatomy, Medical School of Harvard University.

IF we study a number of brains by the section method, or even glance through some good works, such as Dalton's “Atlas" or Fraser's “Morphology of the Brain," which show photographs of brain sections, we cannot fail to observe the great variations in length and shape of the posterior horn of the lateral ventricle. Nowhere in the literature is there sufficient stress laid upon this obviously important characteristic, and although it must in some measure be well known to all anatomists, in only three works could there be found any mention of the fact that the posterior horn varies in length, and no reference to other anomalies which, when fully understood, must be of great clinical importance.

I have recently studied a series of brains in the Medical School of Harvard University, and rarely found what is usually represented as the normal posterior horn. Out of sixty-four adult brains not specially chosen, all grades of variation were met with from the dilated ventricle of hydrocephalus internus to complete occlusions of the posterior horns, and these were numerous. Observations on brains coming, as dissecting-room subjects usually do, from institutions of various

11 R. Jones: Ann. Surg., 1902, xxxv, p. 697. Cotton: Boston Med. AND SURG. Jour., December, 1903, exliv, p. 737 . 